\title{
Global medicinal chemistry and GPCR conference: interview with Stevan Djuric
}

\author{
Stevan Djuric*,1 \\ ${ }^{1}$ Global Pharmaceutical Research \& Development, Abbvie, Inc., North Chicago, IL, USA \\ *Author for correspondence: stevan.w.djuric@abbvie.com
}

\begin{abstract}
Stevan Djuric speaks to Benjamin Walden, Commissioning Editor. Stevan Djuric is head of the global Medicinal Chemistry Leadership Team at AbbVie and is also Vice President of the Discovery Chemistry and Technology organization within their Discovery organization and chemistry outsourcing activities. He spoke at the Global-Medicinal-Chemistry and GPCR summit on the imperative to develop chemistry related technology that can reduce cycle time, cost of goods and improve probability of success. To this end, he discussed his efforts in the chemistry technology area with a focus on integrated synthesis-purification bioassay, and flow photochemistry and high temperature chemistry platforms.
\end{abstract}

First draft submitted: 19 December 2017; Accepted for publication: 4 January 2018; Published online: 27 March 2018

Keywords: drug discovery $\bullet$ encoded library technology $\bullet$ high throughput chemistry $\bullet$ photochemistry

\section{Tell us a little about yourself \& your role at AbbVie}

I am Vice President for AbbVie's Discovery Chemistry Technology organization. The remit of the group is contemporary medicinal design, synthesis activities and new enabling chemistry technology development. This includes advances in the area of high throughput chemistry, high throughput experimentation, DNA encoded libraries, laboratory robotics, flow chemistry techniques such as segmented flow library production, photochemistry and high temperature chemistry.

Can you tell us about the recent work your team has being doing at AbbVie \& how it fits into the wider scheme of drug discovery?

One of our major initiatives, as of late, has been to reduce cycle time for the medicinal chemistry components of the drug discovery process. In this context we have developed (over a 2.5 year period) an integrated platform that achieves synthesis, purification and bioassay of compounds on a multi-milligram scale with a cycle time of $2-3$ days. This compares very favorably with previous cycle times of 7-10 days.

We have just been in your talk on the importance of reducing the drug discovery cycle, could you give a brief rundown of the presentation?

Aside from integrated synthesis/bioassay systems we have, over the last 8 years, developed a chemistry toolkit including flow photochemistry, electrochemistry and high temperature chemistry that allows AbbVie chemists to tap into new reactivity space. AbbVie chemists must make the 'best' molecules - not the easiest ones to make. No molecule should be synthetically inaccessible to them.

What is the rationale behind the accelerated synthesis methods discussed in the talk? What are the key steps in integrating this technology effectively in discovery timeline?

Long cycle times are a well-known problem in drug discovery and development. Our goal is to accelerate the synthesis and testing of molecules utilizing the integration of technology and ensuring management buy-in to the development of new enabling technologies, which we have. Technologies that are developed must have routine utilization within the organization or they are failures. 


\section{What are the main challenges you have encountered in the effective use of these technologies?}

Adoption by the organization, many people are set in their ways and resist change. However, once they see how well things work in a centralized expert service setting (centralized global chemistry technology organization) it often becomes difficult to keep up with demand.

\section{Are there any specific advances in discovery timescale, not mentioned in the talk, that are likely to make an impact in the near future?}

The identification of high quality, validated and small molecule targets is a major current challenge. In many cases, timelines for validation activities whether by biologic methods (CRISPR, KO/KI, shRNA, etc.) or small molecule chemical probes can be lengthy and advances in these areas will be enabling.

\section{What are the next steps for your team \& the wider field?}

In the chemistry technology area, advances in the high throughput experimentation area are helping identify relatively optimized reaction conditions more effectively. We remain interested in robotics and artificial intelligence in the lab and have collaborations in these areas. We continue to look for other methods to accelerate reactions and have been evaluating micro-droplet technology for several years.

\section{Disclaimer}

The opinions expressed in this interview are those of the interviewee and do not necessarily reflect the views of Future Science Ltd.

Financial \& competing interests disclosure

S Djuric is an employee of AbbVie, Inc. The author has no other relevant affiliations or financial involvement with any organization or entity with a financial interest in or financial conflict with the subject matter or materials discussed in the manuscript apart from those disclosed.

No writing assistance was utilized in the production of this manuscript. 